\title{
Australia Day 2016: alcohol-related presentations to emergency departments
}

\author{
Diana Egerton-Warburton ${ }^{1,2}$, Andrew Gosbell ${ }^{3}$, Angela Wadsworth ${ }^{3}$, Daniel M Fatovich ${ }^{4}$, Drew B Richardson ${ }^{5}$
}

A ustralia Day, a national day for commemoration and reflection, has also become associated with high levels of risky drinking. It has been described as "our national day to get drunk and nasty" ${ }^{1}$ Some state-based data indicate that the number of presentations to emergency departments (EDs) related to alcohol use increases on Australia Day, but this has not been quantified at the national level. ${ }^{2,3}$

Information about alcohol-related ED presentations is not routinely collected in patient datasets in Australia. The Australasian College for Emergency Medicine (ACEM) has conducted two point prevalence surveys and one 7-day survey to quantify the level of alcohol harm presenting to Australasian EDs. These surveys have found that one in eight presentations to Australasian EDs during high alcohol hours are alcohol-related, and that the rates in Australia and New Zealand are similar. ${ }^{4,5}$

Our study aimed to quantify the proportion of alcohol-related presentations to Australian EDs on Australia Day, and to compare it with New Zealand EDs. We applied a validated point prevalence snapshot methodology at $11 \mathrm{pm}$ local time on Tuesday, 26 January 2016. The complete methodology for the survey is described in an article on our 2013 snapshot survey of alcohol-related presentations. ${ }^{4}$ ACEM-accredited EDs in New Zealand were invited to participate in the study as a control group, as there were no public holidays or major events in New Zealand on 26 January. The Low Risk Sub-Committee of the Australia Capital Territory Health Human Research and Ethics Committee approved the study (reference, ethlr.13.294), and site-specific governance approval was also obtained as required.

A total of 122 of 131 eligible EDs (93\%) returned data (including 115 in Australia); 386 of 2556 patients in Australian EDs (15.1\%; $95 \%$ confidence interval $[\mathrm{CI}], 13.8-16.6 \%$ ) and 46 of 557 patients in
New Zealand EDs $\left(8.3 \%\right.$; $95 \%$ CI, $6.2-10.9 \%: \chi^{2}$ test, $\left.P<0.001\right)$ had an alcohol-related presentation.

The distribution of alcohol-related presentations was skewed to the left; $0-24$ patients per ED were identified, with a median of 2 (interquartile range, $1-4.5$ ) and a prevalence of $0-75 \%$. Seven large Australian hospitals reported that more than $30 \%$ of their workload involved alcohol-related presentations. Rates varied between states, from $9.5 \%$ in Tasmania to $28 \%$ in the Northern Territory, but every state reported a higher rate than New Zealand. The prevalence in Australia was highest in major referral hospitals $(19.1 \%)$, followed by regional referral (13.2\%) and urban district hospitals $(12.7 \%)$.

Australia Day was associated with higher rates of alcohol-related presentations to EDs than the same day in New Zealand. One in seven presentations being alcohol-related is similar to the weekend presentation rates we found in previous surveys. ${ }^{4,5}$ Funding and time constraints prevented us from conducting a second Australiawide survey on a non-holiday Tuesday. As New Zealand is a neighbouring country without a public holiday on 26 January, it was a reasonable comparator: Australia and New Zealand have similar drinking cultures, and our previous investigations found similar alcohol-related presentation rates to EDs in the two countries. ${ }^{4,5}$

Alcohol-related presentations compromise the safety of clinical staff and other patients, and the effective functioning of the ED. ${ }^{6}$ Our results support government regulations for controlling alcohol availability, cost and promotion, in order to start shifting societal attitudes in Australia and New Zealand away from excessive alcohol consumption.

Acknowledgements: Funding was provided by the Australian Rechabite Foundation.

Competing interests: No relevant disclosures.

(c) 2017 AMPCo Pty Ltd. Produced with Elsevier B.V. All rights reserved.
1 Hagan K, Bucci N. Australia Day: the national day to get drunk and nasty. The Age (Melbourne) 24 Jan 2014. http://www.theage.com.au/victoria/australia-day-thenational-day-to-get-drunk-and-nasty-20140123-31bua. html (accessed Sept 2016).

2 Lloyd B, Matthews S, Livingston M, Jayasekara $\mathrm{H}$. Drinking cultures and social occasions: alcohol harms in the context of major public holidays and cultural events. Melbourne: Turning Point Alcohol and Drug Centre, 2011. https://www.vichealth.vic.gov.au/ /media/ resourcecentre/publicationsandresources/alcohol\% 20misuse/drinking-cultures-social-occasions-report_ public\%20holiday.ashx (accessed Sept 2016).

3 Government of Western Australia. Alcohol and Australia Day celebrations. alcoholthinkagain [website]. 2014. http://alcoholthinkagain.com.au/Alcohol-YourCommunity/Alcohol-and-Australia-Day-Celebrations (accessed Oct 2016).

4 Egerton-Warburton D, Gosbell A, Wadsworth A, et al. Survey of alcohol-related presentations to Australasian emergency departments. Med J Aust 2014; 201: 584-587. https://www.mia.com.au/journal/2014/201/10/survey- alcohol-related-presentations-australasian-emergencydepartments

5 Egerton-Warburton D, Gosbell A, Wadsworth A, et al. Alcohol harm in ED snapshot 2014 [abstract]. Emerg Med Australas 2016; 28 Suppl SI: 13.

6 Egerton-Warburton D, Gosbell A, Wadsworth A, et al. Perceptions of Australasian emergency department staff of the impact of alcohol-related presentations. Med J Aust 2016; 204: 155. https://www.mja.com.au/journal/ 2016/204/4/perceptions-australasian-emergencydepartment-staff-impact-alcohol-related 